PUPT-1696

SLAC-PUB-7452

Imperial/TP/96-97/40

hep-th/9704112

\title{
Intermediate Scalars and the Effective String Model of Black Holes
}

\author{
Igor R. Klebanov, \\ Joseph Henry Laboratories, Princeton University, Princeton, NJ 08544 \\ Arvind Rajaraman, \\ Stanford Linear Accelerator Center, Stanford, CA 94305 \\ and \\ Arkady A. Tseytlin ${ }^{\dagger}$ \\ Blackett Laboratory, Imperial College, London SWr 2BZ, U.K.
}

\begin{abstract}
We consider five-dimensional black holes modeled by D-strings bound to D5-branes, with momentum along the D-strings. We study the greybody factors for the non-minimally coupled scalars which originate from the gravitons and R-R antisymmetric tensor particles polarized along the 5-brane, with one index along the string and the other transverse to the string. These scalars, which we call intermediate, couple to the black holes differently from the minimal and the fixed scalars which were studied previously. Analysis of their fluctuations around the black hole reveals a surprising mixing between these NS-NS and $\mathrm{R}-\mathrm{R}$ scalars. We disentangle this mixing and obtain two decoupled scalar equations. These equations have some new features, and we are able to calculate the greybody factors only in certain limits. The results agree with corresponding calculations in the effective string model provided one of the intermediate scalars couples to an operator of dimension $(1,2)$, while the other to an operator of dimension $(2,1)$. Thus, the intermediate scalars are sensitive probes of the chiral operators in the effective string action.
\end{abstract}

April 1997

$\dagger$ Also at Lebedev Physics Institute, Moscow. 


\section{Introduction}

There has been much progress recently in describing the microstates of black holes through D-brane physics. The Bekenstein-Hawking entropy of certain extremal and nearextremal black holes can be understood through the counting of D-brane microstates [1, 2, [3, [4, 5]. Furthermore, the Hawking radiation and the semi-classical absorption were shown in many cases to agree with the calculation of the corresponding process in the D-brane picture. This was demonstrated for the charged black holes in four and five dimensions that are described by effective string models [6, 0, 8, 9, 10, 11, 12, 13, 14, [15, 16], as well as for the extremal threebranes that admit a direct D-brane description [17,18].

The results mentioned so far refer to minimally coupled scalar fields. Not all scalars, however, are minimally coupled. There are other scalars which couple to the non-trivial vector backgrounds. Examples of these are the 'fixed' scalars considered in [19, 20], which have different cross-sections from the minimally coupled scalars. In the $D=5$ black hole background there are two specific fixed scalars, which mix with each other and with the gravitational perturbations [20]. Recently, the complexities of this mixing were disentangled in [21]. The greybody factors calculated from the diagonalized equations of motion were found to be of the form obtained earlier in [20]: in the effective string model such greybody factors are reproduced by operators of dimension $(2,2)$. This poses a puzzle, since the effective string action derived in [20] also contains couplings to dimension $(1,3)$ and $(3,1)$ operators which produce greybody factors of a different form. Thus, it is of special interest to study other situations in which chiral operators appear in the effective string couplings. This will be the subject of the present paper.

We will be concerned with yet a third type of scalars, which we call intermediate, first considered in [20]. This type is different from both the minimally coupled and the fixed scalars. The intermediate scalars originate from the fields $A_{5}^{i}$ (denoted by $h_{5 i}$ in [20]) and $B_{5 i}$, i.e. the gravitons and the R-R 2-form particles polarized along the 5-brane, with one index pointing along the string and the other transversely to the string. In this paper, we will calculate the semi-classical absorption cross-sections of the intermediate scalars and compare them with the effective string model predictions.

In Section 2, after presenting the setup, we derive the classical equations of motion for the intermediate scalars in the $D=5$ black hole background (an alternative derivation based on the 6-dimensional theory will be presented in the Appendix). This turns out to be quite nontrivial due to a mixing between $A_{5}^{i}$ and $B_{5 i}$. In Section 3 we propose a coupling for these scalars in the effective string model of the black hole. Part of this coupling term is not present in the standard Nambu-type D-string action. It turns out that requiring the scalars to couple to operators of a given dimension on the world sheet is a very restrictive guiding principle. We find that the necessary operators are of dimensions $(1,2)$ and $(2,1)$ and then calculate the resulting cross-sections as predicted by the effective string model. 
Finally, in Section 4 we compare the absorption cross-sections derived by semi-classical considerations to the cross-sections predicted by the string. The classical equations of motion are complicated and we are only able to solve for the cross-sections in various limits. In every case that we can treat analytically, there is exact agreement between the semi-classical gravity and the effective string. This is evidence that the effective string model reproduces the dynamics of the intermediate scalars. However, our inability to solve for the general semi-classical greybody factor leaves the question of the complete agreement open.

\section{Derivation of the Equations of Motion}

As in [20] we start with the action of the $D=10$ type IIB supergravity reduced to 5 dimensions. The relevant part of it is

$$
\begin{aligned}
S_{5}= & \frac{1}{2 \kappa_{5}^{2}} \int d^{5} x \sqrt{g}\left[R-\frac{4}{3}\left(\partial_{\mu} \phi_{5}\right)^{2}-\frac{1}{4} G^{p l} G^{q n}\left(\partial_{\mu} G_{p q} \partial^{\mu} G_{l n}+\sqrt{G} \partial_{\mu} B_{p q} \partial^{\mu} B_{l n}\right)\right. \\
& \left.-\frac{1}{4} e^{-\frac{4}{3} \phi_{5}} G_{p q} F_{\mu \nu}^{p} F_{\mu \nu}^{q}-\frac{1}{4} e^{\frac{2}{3} \phi_{5}} \sqrt{G} G^{p q} H_{\mu \nu p} H_{\mu \nu q}-\frac{1}{12} e^{\frac{4}{3} \phi_{5}} \sqrt{G} H_{\mu \nu \lambda}^{2}+\ldots\right],
\end{aligned}
$$

where $\mu, \nu, \ldots=0,1,2,3,4 ; p, q, \ldots=5,6,7,8,9 . \quad \phi_{5}$ is the 5 -d dilaton and $G_{p q}$ is the metric of internal 5 -torus,

$$
\phi_{5} \equiv \phi_{10}-\frac{1}{4} G=\phi_{6}-\frac{1}{2} \lambda, \quad G=\operatorname{det} G_{p q}
$$

and $B_{p q}$ are the internal components of the R-R 2-tensor. $F_{\mu \nu}^{p}$ is the field strength of the Kaluza-Klein vectors $A_{\mu}^{p}$. It will be crucial in what follows that $H_{\mu \nu p}$ and $H_{\mu \nu \lambda}$ are given explicitly by (see, e.g., [22])

$$
\begin{gathered}
H_{\mu \nu p}=F_{\mu \nu p}-B_{p q} F_{\mu \nu}^{q}, \quad F_{p}=d B_{p}, \quad F^{p}=d A^{p}, \\
H_{\mu \nu \lambda}=\partial_{\mu} B_{\nu \lambda}-\frac{1}{2} A_{\mu}^{p} F_{\nu \lambda p}-\frac{1}{2} B_{\mu p} F_{\nu \lambda}^{p}+\text { cyclic permutations },
\end{gathered}
$$

where $B_{\mu p}$ and $B_{\mu \nu}$ differ from the $D=10$ components of the R-R 2-form field by terms proportional to $A_{\mu}^{p}$. The 'shifts' in these field strengths vanish for the $D=5$ black hole backgrounds which correspond to bound states of RR-charged 5-branes and strings with momentum flow. For such black holes, $B_{p q}=0$, the vector fields $A^{p}$ and $B_{p}$ have electric backgrounds, while $H_{\mu \nu \lambda}$ has a magnetic one (we shall assume that the electric charges $Q_{K p}$ and $Q^{p}$ corresponding to the vectors $A^{p}$ and $B_{p}$ have only the $p=5$ component). However, in general the field strength shifts in (2.2) are important for the discussion of 
perturbations. We will argue, in fact, that while the shift in $H_{\mu \nu \lambda}$ does not contribute in the present case, the shift in $H_{\mu \nu p}$ will lead to a mixing between perturbations of $G_{p q}$ and $B_{p q}$ for $p=5$ and $q=i$ ( 5 is the direction of the string and $i=6,7,8,9$ label the directions of $T^{5}$ orthogonal to the string).

The 5-dimensional charged black hole metric is [23, 24, [4]

$$
\begin{gathered}
d s_{5}^{2}=g_{m n} d x^{m} d x^{n}+d s_{3}^{2}=-h \mathcal{H}^{-2} d t^{2}+h^{-1} \mathcal{H} d r^{2}+r^{2} \mathcal{H} d \Omega_{3}^{2} \\
h=1-\frac{r_{0}^{2}}{r^{2}}, \quad \mathcal{H} \equiv\left(H_{n} H_{1} H_{5}\right)^{1 / 3}, \quad \sqrt{g}=r^{3}\left(H_{n} H_{1} H_{5}\right)^{1 / 3} \\
H_{1}=1+\frac{\hat{Q}}{r^{2}}, \quad H_{5}=1+\frac{\hat{P}}{r^{2}}, \quad H_{n}=1+\frac{\hat{Q}_{K}}{r^{2}}
\end{gathered}
$$

where $\hat{Q}=\sqrt{Q^{2}+\frac{1}{4} r_{0}^{4}}-\frac{1}{2} r_{0}^{2}$, etc. The background values of the internal metric and the dilaton are (see [20] for more details)

$$
\begin{gathered}
\left(d s_{10}^{2}\right)_{T^{5}}=G_{p q} d x^{p} d x^{q}=e^{2 \nu_{5}} d x_{5}^{2}+e^{2 \nu}\left(d x_{6}^{2}+d x_{7}^{2}+d x_{8}^{2}+d x_{9}^{2}\right), \\
\nu_{5}=-2 \phi_{5} \equiv \lambda, \quad e^{2 \lambda}=\frac{H_{n}}{\left(H_{1} H_{5}\right)^{1 / 2}} .
\end{gathered}
$$

It is useful to choose the following parametrization for the full (background plus perturbation) internal metric

$$
\begin{gathered}
G_{p q}=e^{2 \nu}\left(\begin{array}{cc}
e^{2 \lambda-2 \nu}+e^{2 \nu} A_{5}^{i} A_{5}^{i} & A_{5}^{i} \\
A_{5}^{j} & \delta_{i j}
\end{array}\right), \quad \sqrt{G}=e^{\lambda+4 \nu}, \\
G^{p q}=e^{-2 \lambda}\left(\begin{array}{cc}
1 & -A_{5}^{i} \\
-A_{5}^{j} & e^{2 \lambda-2 \nu} \delta^{i j}+A_{5}^{i} A_{5}^{j}
\end{array}\right),
\end{gathered}
$$

For the present discussion of the 'off-diagonal' perturbations the fluctuations of $\phi_{5}$, as well as those of $\sqrt{G}$, can be ignored. Therefore, we concentrate on the dependence on $A_{5}^{i}$ and $B_{5 i}$ and do not keep track of other scalar perturbations which were already discussed in [20].

The $D=5$ scalars $A_{5}^{i}$ and $B_{5 i}$ originate from the $M=5$ components of the KK vector $A_{M}^{i}$ and the vector component $B_{M i}$ of the R-R 2-tensor in type IIB supergravity reduced to 6 dimensions. An alternative derivation of the equations for the $A_{5}^{i}$ and $B_{5 i}$ perturbations, which directly uses the $D=6$ theory, will be presented in the Appendix.

The relevant terms in the $D=5$ action ard 1

$$
S_{5}=\frac{1}{2 \kappa_{5}^{2}} \int d^{5} x \sqrt{g}\left[-\frac{1}{2} e^{-2 \lambda+2 \nu} \partial_{\mu} A_{5}^{i} \partial^{\mu} A_{5}^{i}-\frac{1}{2} e^{-2 \lambda+2 \nu} \partial_{\mu} B_{5 i} \partial^{\mu} B_{5 i}\right.
$$

1 The $\mu, \nu$ indices are always contracted using the curved 5-dimensional metric and assuming that $F_{\mu \nu} F_{\mu \nu} \equiv F_{\mu \nu} F^{\mu \nu}$, etc. The repeated $i, j$-indices are summed with $\delta_{i j}$ with no extra factors (all factors in $5, i$ directions are given explicitly). 


$$
\begin{gathered}
-\frac{1}{4} e^{\frac{2}{3} \lambda+2 \nu}\left[F_{\mu \nu}^{i} F_{\mu \nu}^{i}+2 F_{\mu \nu}^{5} F_{\mu \nu}^{i} A_{5}^{i}+F_{\mu \nu}^{5} F_{\mu \nu}^{5}\left(e^{2 \lambda-2 \nu}+A_{5}^{i} A_{5}^{i}\right)\right] \\
\left.-\frac{1}{4} e^{-\frac{4}{3} \lambda+4 \nu}\left[H_{\mu \nu 5} H_{\mu \nu 5}-2 H_{\mu \nu i} H_{\mu \nu 5} A_{5}^{i}+H_{\mu \nu i} H_{\mu \nu j}\left(e^{2 \lambda-2 \nu} \delta^{i j}+A_{5}^{i} A_{5}^{j}\right)\right]\right]
\end{gathered}
$$

where

$$
H_{\mu \nu 5}=F_{\mu \nu 5}-B_{5 i} F_{\mu \nu}^{i}, \quad H_{\mu \nu i}=F_{\mu \nu i}+B_{5 i} F_{\mu \nu}^{5} .
$$

Here only $F_{\mu \nu 5}$ and $F_{\mu \nu}^{5}$ have background values, which we denote by $\tilde{F}$,

$$
e^{\frac{8}{3} \lambda} \sqrt{g}\left(\tilde{F}^{5}\right)^{0 r}=2 Q_{K}, \quad e^{-\frac{4}{3} \lambda+4 \nu} \sqrt{g}\left(\tilde{F}_{5}\right)^{0 r}=2 Q
$$

so that

$$
\tilde{F}_{\mu \nu}^{5}=\frac{Q_{K}}{Q} e^{-4 \lambda+4 \nu} \tilde{F}_{\mu \nu 5}
$$

As a result, we may integrate out $F_{\mu \nu i}$ or all of $H_{\mu \nu i}$ easily. This gives 2

$$
-\frac{1}{4} e^{-\frac{4}{3} \lambda+4 \nu}\left(-e^{-2 \lambda+2 \nu} \tilde{F}_{\mu \nu 5} \tilde{F}_{\mu \nu 5} A_{5}^{i} A_{5}^{i}\right) .
$$

To show this it is crucial that $\tilde{F}_{\mu \nu}^{5}$ has only the electric component and depends only on $r$, and that the scalar perturbations depend only on $r$ and $t$. This is similar to what happens in the fixed scalar case [19,20].

The mixing that contributes a new term is $\tilde{F}_{\mu \nu 5} B_{5 i} F_{\mu \nu}^{i}$ which comes from the $H_{\mu \nu 5}^{2}$ term. 3 The relevant vector-scalar terms are

$$
-\frac{1}{4} e^{\frac{2}{3} \lambda} e^{2 \nu}\left[F_{\mu \nu}^{i} F_{\mu \nu}^{i}+2 \tilde{F}_{\mu \nu}^{5} F_{\mu \nu}^{i} A_{5}^{i}+\tilde{F}_{\mu \nu}^{5} \tilde{F}_{\mu \nu}^{5} A_{5}^{i} A_{5}^{i}-2 e^{-2 \lambda+2 \nu} \tilde{F}_{\mu \nu 5} B_{5 i} F_{\mu \nu}^{i}\right]
$$

It remains to integrate out $F_{\mu \nu}^{i}$. One should actually integrate over the corresponding gauge potential, but since the background is electric and static, and the scalars depend only on $r$ and $t$, this is equivalent to just solving for the field strength.

2 The $H_{i} H_{i} h h$ term is of subleading order being quartic in the fluctuations.

3 One way to see why the mixing terms inside $H_{\mu \nu \lambda}$ in (2.1) and (2.2) do not contribute is to dualize $B_{\mu \nu}$ into a vector, $V_{\mu}$. The resulting terms in the action will have the following structure: $\int d^{5} x\left[-\frac{1}{4} \sqrt{g} e^{-\frac{4}{3} \phi_{5}} G^{-1 / 2} F_{\mu \nu}^{2}(V)+\epsilon^{\mu \nu \lambda \sigma \kappa} V_{\mu} F_{\nu \lambda p} F_{\sigma \kappa}^{p}\right]$. The three vectors, $V_{\mu}, A_{\mu 5}, A_{\mu}^{5}$, have electric backgrounds with charges $P, Q, Q_{K}$ respectively. The trilinear Chern-Simons-type term produces a non-zero contribution in the gaussian approximation only if the two fluctuation fields have indices different from 0 and $r$, which are the directions of the electric background of the third field in the product. This means that the Chern-Simons-type term does not mix the 'electric' perturbations of the fields, but it is the 'electric' perturbations of the vectors that couple to the off-diagonal scalars we discuss. 
Adding the $A_{5}^{i} A_{5}^{i}$ term already obtained in (2.8), we get

$$
\begin{aligned}
& -\frac{1}{4} e^{-\frac{2}{3} \lambda+2 \nu}\left(-\left[\tilde{F}_{\mu \nu}^{5} A_{5}^{i}-e^{-2 \lambda+2 \nu} \tilde{F}_{\mu \nu 5} B_{5 i}\right]^{2}\right. \\
& \left.+\tilde{F}_{\mu \nu}^{5} \tilde{F}_{\mu \nu}^{5} A_{5}^{i} A_{5}^{i}-e^{-4 \lambda+4 \nu} \tilde{F}_{\mu \nu 5} \tilde{F}_{\mu \nu 5} A_{5}^{i} A_{5}^{i}\right)
\end{aligned}
$$

We can simplify this expression using (2.7):

$$
\begin{gathered}
\frac{1}{4} e^{-\frac{2}{3} \lambda+2 \nu}\left(e^{-4 \lambda+4 \nu} \tilde{F}_{\mu \nu 5} \tilde{F}_{\mu \nu 5}\left(A_{5}^{i} A_{5}^{i}+B_{5 i} B_{5 i}\right)-2 e^{-2 \lambda+2 \nu} \tilde{F}_{\mu \nu}^{i} \tilde{F}_{\mu \nu 5} A_{5}^{i} B_{5 i}\right) \\
=\frac{1}{4} e^{-\frac{14}{3} \lambda+6 \nu} \tilde{F}_{\mu \nu 5} \tilde{F}_{\mu \nu 5}\left(A_{5}^{i} A_{5}^{i}+B_{5 i} B_{5 i}-2 \frac{Q_{K}}{Q} e^{-2 \lambda+2 \nu} A_{5}^{i} B_{5 i}\right) .
\end{gathered}
$$

The novelty is the mixing term in the brackets

$$
-2 \frac{Q_{K}}{Q} e^{-2 \lambda+2 \nu} A_{5}^{i} B_{5 i}=-2 \frac{Q_{K} H_{1}}{Q H_{n}} A_{5}^{i} B_{5 i}
$$

which is thus present for arbitrary non-vanishing values of $P, Q$ and $Q_{K}$.

Remarkably, the full $A_{5}^{i}, B_{5 i}$ scalar action with the kinetic terms included can be diagonalized in terms of the fields $\xi_{i}$ and $\eta_{i}$ defined by

$$
\eta_{i}=\frac{1}{\sqrt{2}}\left(A_{5}^{i}+B_{5 i}\right), \quad \xi_{i}=\frac{1}{\sqrt{2}}\left(A_{5}^{i}-B_{5 i}\right)
$$

With these definitions,

$$
\begin{gathered}
S_{5}=\frac{1}{2 \kappa_{5}^{2}} \int d^{5} x \sqrt{g}\left(-\frac{1}{2} e^{-2 \lambda+2 \nu}\left[\left(\partial_{\mu} \xi_{i}\right)^{2}+\left(\partial_{\mu} \eta_{i}\right)^{2}\right]\right. \\
\left.+\frac{1}{4} e^{-\frac{14}{3} \lambda+6 \nu} \tilde{F}_{\mu \nu 5} \tilde{F}_{\mu \nu 5}\left[\left(1+\frac{Q_{K}}{Q} e^{-2 \lambda+2 \nu}\right) \xi_{i}^{2}+\left(1-\frac{Q_{K}}{Q} e^{-2 \lambda+2 \nu}\right) \eta_{i}^{2}\right]\right) .
\end{gathered}
$$

Rescaling the fields to eliminate the background-dependent factors $e^{-2 \lambda+2 \nu}$ in the kinetic parts, we arrive at the following decoupled equations (we shall use the same notation, $\xi_{i}$ and $\eta_{i}$, for the redefined fields, $e^{-\lambda+\nu} \xi_{i}$ and $\left.e^{-\lambda+\nu} \eta_{i}\right)$

$$
\begin{aligned}
& {\left[h r^{-3} \frac{d}{d r}\left(h r^{3} \frac{d}{d r}\right)+\omega^{2} H_{1} H_{5} H_{n}-M_{\xi}\right] \xi_{i}=0} \\
& {\left[h r^{-3} \frac{d}{d r}\left(h r^{3} \frac{d}{d r}\right)+\omega^{2} H_{1} H_{5} H_{n}-M_{\eta}\right] \eta_{i}=0}
\end{aligned}
$$

where

$$
M_{\xi}=M_{\lambda-\nu}+M_{+}, \quad M_{\eta}=M_{\lambda-\nu}+M_{-},
$$




$$
\begin{gathered}
M_{\lambda-\nu}=h e^{\lambda-\nu} r^{-3} \frac{d}{d r}\left(r^{3} h \frac{d}{d r} e^{-\lambda+\nu}\right) \\
M_{ \pm}=\frac{4 Q^{2}}{r^{6} H_{1}^{2}}\left(1 \pm \frac{Q_{K} H_{1}}{Q H_{n}}\right) h
\end{gathered}
$$

Somewhat surprisingly, all the dependence on $P$ disappears from the "mass" terms since

$$
e^{-\lambda+\nu}=\left(H_{1} / H_{n}\right)^{1 / 2}
$$

so that

$$
\begin{aligned}
M_{\lambda-\nu} & =\frac{\left(\hat{Q}_{K}-\hat{Q}\right)\left(r^{2}-r_{0}^{2}\right)}{r^{4}\left(r^{2}+\hat{Q}_{K}\right)^{2}\left(r^{2}+\hat{Q}\right)^{2}}\left[\left(\hat{Q}+3 \hat{Q}_{K}+2 r_{0}^{2}\right) r^{4}\right. \\
& \left.+\left(4 \hat{Q} \hat{Q}_{K}+\hat{Q} r_{0}^{2}-\hat{Q}_{K} r_{0}^{2}\right) r^{2}-2 \hat{Q} \hat{Q}_{K} r_{0}^{2}\right] .
\end{aligned}
$$

In the extremal limit, $r_{0}=0, \hat{Q}=Q, \hat{P}=P, \hat{Q}_{K}=Q_{K}$, the resulting "mass" terms are found to be

$$
\begin{gathered}
M_{\xi}=\frac{8 Q^{2} Q_{K}^{2}+8 Q Q_{K}\left(Q+Q_{K}\right) r^{2}+\left(3 Q^{2}+2 Q Q_{K}+3 Q_{K}^{2}\right) r^{4}}{r^{2}\left(r^{2}+Q_{K}\right)^{2}\left(r^{2}+Q\right)^{2}} \\
M_{\eta}=\frac{3\left(Q-Q_{K}\right)^{2} r^{2}}{\left(r^{2}+Q_{K}\right)^{2}\left(r^{2}+Q\right)^{2}} .
\end{gathered}
$$

They have the following asymptotics

$$
\begin{gathered}
r \rightarrow 0: \quad M_{\xi}=\frac{8}{r^{2}}, \quad M_{\eta}=0 \\
r \rightarrow \infty: \quad M_{\xi}=\frac{3 Q^{2}+2 Q Q_{K}+3 Q_{K}^{2}}{r^{6}}, \quad M_{\eta}=\frac{3\left(Q-Q_{K}\right)^{2}}{r^{6}} .
\end{gathered}
$$

At the horizon $\eta_{i}$ behaves as the $l=0$ partial wave of a minimally coupled scalar. $\xi_{i}$, on the other hand, behaves as the $l=2$ partial wave, which is the behavior previously encountered for the fixed scalars [20,21]. The expressions (2.14) can be simplified if $Q \gg Q_{K}$,

$$
\begin{gathered}
M_{\xi}=\frac{Q^{2}\left(8 Q_{K}^{2}+8 Q_{K} r^{2}+3 r^{4}\right)}{r^{2}\left(r^{2}+Q_{K}\right)^{2}\left(r^{2}+Q\right)^{2}}, \\
M_{\eta}=\frac{3 Q^{2} r^{2}}{\left(r^{2}+Q_{K}\right)^{2}\left(r^{2}+Q\right)^{2}} .
\end{gathered}
$$

Note that, for $Q_{K}=0$,

$$
M_{\eta}=M_{\xi}=\frac{3 Q^{2}}{r^{2}\left(r^{2}+Q\right)^{2}} .
$$

Thus, as one switches on $Q_{K}$, there is a remarkable jump from the $l=1$ to the $l=0$ or $l=2$ behaviors near the horizon. 


\section{Absorption in the Effective String Model}

In the previous section we found a surprising mixing between the off-diagonal components of the Kaluza-Klein scalars $A_{5}^{i}$ and the internal components $B_{5 i}$ of the R-R 2-tensor. In this section we discuss this mixing from the effective string point of view, and show what it implies about the greybody factors.

First, we have to write down the lowest-dimension couplings to the effective string for the fields in question. In [20] the scalar fields $A_{5}^{i}$ were included, but the components $B_{5 i}$ of the R-R field were omitted. In fact, as the discussion of the gravitational perturbations shows, these two field mix and one should keep both of them. The simplest assumption that one usually makes is that the effective string action is the same as the D-string action with a rescaled tension. The necessary terms in the action are then

$$
S=-T_{\mathrm{eff}} \int d^{2} \sigma\left(\sqrt{-\gamma}-\hat{B}_{05}\right)
$$

where

$$
\gamma_{a b}=G_{\mu \nu}(X) \partial_{a} X^{\mu} \partial_{b} X^{\nu}, \quad \hat{B}_{a b}=B_{\mu \nu}(X) \partial_{a} X^{\mu} \partial_{b} X^{\nu} .
$$

The leading order couplings are found to be

$$
-\frac{T_{\mathrm{eff}}}{2}\left[A_{5}^{i}\left(\partial_{+}+\partial_{-}\right) X^{i}+B_{5 i}\left(\partial_{+}-\partial_{-}\right) X^{i}\right]=-\frac{T_{\mathrm{eff}}}{\sqrt{2}}\left[\xi_{i} \partial_{-} X^{i}+\eta_{i} \partial_{+} X^{i}\right],
$$

where the same mixtures of the fields naturally emerge as the ones needed in the effective field theory (GR) calculation, (2.10). We see that these mixtures couple to operators of dimension $(0,1)$ and $(1,0)$ respectively. Clearly, these couplings do not contribute to absorption. Expanding further we find the term

$$
-\frac{T_{\mathrm{eff}}}{4} A_{5}^{i}\left[\partial_{-} X^{i}\left(\partial_{+} X\right)^{2}+\partial_{+} X^{i}\left(\partial_{-} X\right)^{2}\right]
$$

whose natural supersymmetric completion is

$$
-\frac{T_{\mathrm{eff}}}{4} A_{5}^{i}\left[\partial_{-} X^{i} T_{++}^{t o t}+\partial_{+} X^{i} T_{--}^{t o t}\right]
$$

with $T^{\text {tot }}$ including the fermionic contribution as well. It is interesting that, using this coupling in the case $Q_{K}=0$, we find the greybody factor which exactly agrees with the GR result. So, for $Q_{K}=0$ (the non-chiral case) we may just use the coupling stated in [20] and arrive at complete agreement with the semi-classical calculation.

The structure of the action is less clear for $Q_{K}>0$. While we do not readily see a cubic coupling for $B_{5 i}$, we will add it by hand to enforce the principle that $\xi_{i}$ and $\eta_{i}$ 
couple to operators of a given dimension. With this assumption, the terms that arise in the effective string action are

$$
\delta S=-\frac{T_{\mathrm{eff}} \sqrt{2}}{4} \int d^{2} \sigma\left[\eta_{i} \partial_{-} X^{i} T_{++}^{t o t}+\xi_{i} \partial_{+} X^{i} T_{--}^{t o t}\right]
$$

Using the action (3.1), let us now derive the effective string absorption cross-section for $\eta_{i}$. The absorption cross-section is due to processes $\eta_{i} \rightarrow L+L+R$ and $\eta_{i}+L \rightarrow L+R$ ( $L$ and $R$ stand for the left-moving and right-moving modes on the string). The matrix element between properly normalized states, including the kinetic term normalization factor $\kappa_{5} \sqrt{2}$ for $\eta_{i}$ (see $(2.11)$ ), is found to be

$$
\frac{2 \kappa_{5}}{\sqrt{T_{\text {eff }}}} \sqrt{\frac{q_{1} p_{1} p_{2}}{\omega}}
$$

Adding up the absorption rates for the two processes gives (see [20] for details of analogous cross-section computations)

$$
\begin{aligned}
& \frac{3 \kappa_{5}^{2} L_{\mathrm{eff}}}{2 \pi T_{\mathrm{eff}}} \frac{1}{1-e^{-\frac{\omega}{2 T_{R}}}} \int_{-\infty}^{\infty} d p_{1} d p_{2} \delta\left(p_{1}+p_{2}-\frac{\omega}{2}\right) \frac{p_{1}}{1-e^{-\frac{p_{1}}{T_{L}}}} \frac{p_{2}}{1-e^{-\frac{p_{2}}{T_{L}}}} \\
= & \frac{\kappa_{5}^{2} L_{\mathrm{eff}}}{32 \pi T_{\mathrm{eff}}} \frac{\omega}{\left(1-e^{-\frac{\omega}{2 T_{L}}}\right)\left(1-e^{-\frac{\omega}{2 T_{R}}}\right)}\left(\omega^{2}+16 \pi^{2} T_{L}^{2}\right) .
\end{aligned}
$$

The values of the parameters in the effective string model have been fixed in [25, 10, 20],

$$
\kappa_{5}^{2} L_{\mathrm{eff}}=4 \pi^{3} r_{1}^{2} r_{5}^{2}, \quad T_{\mathrm{eff}}=\frac{1}{2 \pi r_{5}^{2}}
$$

where

$$
r_{1}^{2} \equiv \hat{Q}, \quad r_{5}^{2} \equiv \hat{P}
$$

Note that this effective string tension is the tension of the D-string divided by $n_{5}$, the number of 5-branes. This value of the tension is necessary for agreement with the entropy of near-extremal 5-branes [25], as well as for the agreement of the fixed-scalar cross-section for $r_{1}=r_{5}$ [20]. In this paper we will show that it also leads to agreement of the absorption cross-sections for the scalars $\eta_{i}$ and $\xi_{i}$.

Using (3.3), (3.4), and the detailed balance, we find that the absorption cross-section for $\eta_{i}$ is

$$
\sigma_{\eta}(\omega)=\frac{\pi^{3} r_{1}^{2} r_{5}^{4}}{4} \frac{\omega\left(e^{\frac{\omega}{T_{H}}}-1\right)}{\left(e^{\frac{\omega}{2 T_{L}}}-1\right)\left(e^{\frac{\omega}{2 T_{R}}}-1\right)}\left(\omega^{2}+16 \pi^{2} T_{L}^{2}\right)
$$


After analogous steps, the absorption cross-section for $\xi_{i}$ is found to be

$$
\sigma_{\xi}(\omega)=\frac{\pi^{3} r_{1}^{2} r_{5}^{4}}{4} \frac{\omega\left(e^{\frac{\omega}{T_{H}}}-1\right)}{\left(e^{\frac{\omega}{2 T_{L}}}-1\right)\left(e^{\frac{\omega}{2 T_{R}}}-1\right)}\left(\omega^{2}+16 \pi^{2} T_{R}^{2}\right)
$$

In the next section we will check these greybody factors against semi-classical effective field theory calculations. We will need the following expressions for the temperatures [10],

$$
T_{L}=\frac{r_{0} e^{\sigma}}{2 \pi r_{1} r_{5}}, \quad T_{R}=\frac{r_{0} e^{-\sigma}}{2 \pi r_{1} r_{5}}, \quad \frac{2}{T_{H}}=\frac{1}{T_{L}}+\frac{1}{T_{R}}
$$

where $\sigma$ is defined by

$$
r_{n}^{2}=r_{0}^{2} \sinh ^{2} \sigma, \quad r_{n}^{2} \equiv \hat{Q}_{K} .
$$

This may be solved with the result,

$$
e^{ \pm 2 \sigma}=1+\frac{2}{r_{0}^{2}}\left(r_{n}^{2} \pm Q\right)
$$

Under $Q_{K} \rightarrow-Q_{K}$, we therefore find that $\sigma \rightarrow-\sigma$, which implies that $T_{L}$ and $T_{R}$ are interchanged. This transformation reverses the momentum flow along the string, so that the operators of dimension $(1,2)$ and $(2,1)$, and therefore $\xi_{i}$ and $\eta_{i}$, are interchanged. The classical equations for $\xi_{i}$ and $\eta_{i}$, (2.12), (2.13), are also interchanged under $Q_{K} \rightarrow-Q_{K}$. This is the first, and very important, consistency check between the effective string and the semi-classical descriptions of the intermediate scalars.

\section{Comparison with Semiclassical Greybody Factors}

In this section we carry out a number of calculations which indicate agreement, at least in various limits, between the semi-classical cross-sections and those in the effective string model. First we discuss the case $Q_{K}=0$ where the classical calculation is the easiest. Then we address various limits of the $Q_{K}>0$ case.

\section{1. $Q_{K}=0$}

Here we consider the case $r_{n}^{2}=0$ (i.e. $Q_{K}=0$ ), where $\eta_{i}$ and $\xi_{i}$ satisfy identical equations (2.12), 2.16). Since here $T_{L}=T_{R}$, the two effective string greybody factors are also the same, and they will turn out to be identical to the semi-classical ones.

The non-extremal equation satisfied by both $\eta_{i}$ and $\xi_{i}$ is (for $r_{0} \ll r_{1}, r_{5}$ )

$$
\left[h r^{-3} \partial_{r}\left(h r^{3} \partial_{r}\right)+f(r) \omega^{2}-3 h \frac{r_{1}^{4}}{r^{2}\left(r_{1}^{2}+r^{2}\right)^{2}}+h \frac{r_{0}^{2} r_{1}^{2}\left(r_{1}^{2}+2 r^{2}\right)}{r^{4}\left(r_{1}^{2}+r^{2}\right)^{2}}\right] R=0
$$


where we set $\eta_{i}, \xi_{i}=R(r) e^{i \omega t}$, and

$$
h(r)=1-\frac{r_{0}^{2}}{r^{2}}, \quad f(r)=\left(1+\frac{r_{1}^{2}}{r^{2}}\right)\left(1+\frac{r_{5}^{2}}{r^{2}}\right) .
$$

In the near region $\left(r \ll r_{1}, r_{5}\right)$ we find, in terms of the variable $z=h(r)$,

$$
\left[z \partial_{z}\left(z \partial_{z}\right)+D+\frac{C}{(1-z)}+\frac{E}{(1-z)^{2}}\right] R=0
$$

where

$$
D=-\frac{1}{4}, \quad C=\frac{\omega^{2} r_{1}^{2} r_{5}^{2}}{4 r_{0}^{2}}+1, \quad E=-\frac{3}{4} .
$$

This may be reduced to a hypergeometric equation by a substitution of the form

$$
R=z^{\alpha}(1-z)^{\beta} F(z) \text {. }
$$

After some algebra we find that, if $\alpha$ and $\beta$ satisfy

$$
E+\beta(\beta-1)=0, \quad \alpha^{2}+D+C+E=0,
$$

then the equation for $F(z)$ becomes

$$
z(1-z) \frac{d^{2} F}{d z^{2}}+[(2 \alpha+1)(1-z)-2 \beta z] \frac{d F}{d z}-\left[(\alpha+\beta)^{2}+D\right] F=0,
$$

which is the hypergeometric equation. In general, the solution to

$$
z(1-z) \frac{d^{2} F}{d z^{2}}+[C-(1+A+B) z] \frac{d F}{d z}-A B F=0
$$

which satisfies $F(0)=1$, is the hypergeometric function $F(A, B ; C ; z)$. Thus, the solution in the inner region is

$$
R_{I}=z^{\alpha}(1-z)^{\beta} F(\alpha+\beta+i \sqrt{D}, \alpha+\beta-i \sqrt{D} ; 1+2 \alpha ; z)
$$

where

$$
\beta=-\frac{1}{2}, \quad \alpha=-i \frac{\omega r_{1} r_{5}}{2 r_{0}}=-i \frac{\omega}{4 \pi T} .
$$

In the last equation we used the fact that, for $r_{n}=0$,

$$
T=T_{L}=T_{R}=T_{H}=\frac{r_{0}}{2 \pi r_{1} r_{5}}
$$

Using the asymptotics of the hypergeometric functions for $z \rightarrow 1$, we find that, for large $r$,

$$
R_{I} \rightarrow \frac{r}{r_{0}} E,
$$


where

$$
E=\frac{\Gamma\left(1-i \frac{\omega}{2 \pi T}\right)}{\Gamma\left(2-i \frac{\omega}{4 \pi T}\right) \Gamma\left(1-i \frac{\omega}{4 \pi T}\right)} .
$$

In the middle region $\left(r_{0} \ll r \ll 1 / \omega\right)$ the approximate solution is

$$
R_{I I} \approx E \frac{r_{1}}{r_{0}}\left(1+\frac{r_{1}^{2}}{r^{2}}\right)^{-1 / 2}
$$

In the outer region, the dominant solution, which matches to the asymptotic form in region II, is

$$
R_{I I I}=2 A \rho^{-1} J_{1}(\rho), \quad \rho=\omega r .
$$

By matching we find that

$$
A=E \frac{r_{1}}{r_{0}} .
$$

The absorption cross-section may now be obtained using the method of fluxes (see, e.g., [10,20] and references therein). The flux per unit solid angle is

$$
\mathcal{F}=\frac{1}{2 i}\left(R^{*} h r^{3} \partial_{r} R-\text { c.c. }\right) \text {. }
$$

The absorption probability is the ratio of the incoming flux at the horizon to the incoming flux at infinity,

$$
P=\frac{\mathcal{F}_{\text {horizon }}}{\mathcal{F}_{\infty}^{\text {incoming }}}=\frac{\pi \omega^{3}}{2} r_{1} r_{5} r_{0}|A|^{-2} .
$$

The absorption cross-section is related to the s-wave absorption probability by

$$
\sigma_{\mathrm{abs}}=\frac{4 \pi}{\omega^{3}} P=\frac{2 \pi^{2} r_{0}^{3} r_{5}}{r_{1}}|E|^{-2}
$$

Thus,

$$
\sigma_{\mathrm{abs}}=\frac{2 \pi^{2} r_{0}^{3} r_{5}}{r_{1}} x\left(1+x^{2}\right) \frac{e^{2 \pi x}+1}{e^{2 \pi x}-1}
$$

where

$$
x=\frac{\omega}{4 \pi T}=\frac{\omega r_{1} r_{5}}{2 r_{0}}
$$

It follows that

$$
\sigma_{\mathrm{abs}}=\frac{\pi^{3}}{4} r_{1}^{2} r_{5}^{4} \frac{e^{\frac{\omega}{2 T}}+1}{e^{\frac{\omega}{2 T}}-1} \omega\left(\omega^{2}+16 \pi^{2} T^{2}\right)
$$

This is in exact agreement with the cross-sections (3.5) and (3.6) derived in the effective string model! In particular, the agreement of the overall normalization provides new evidence in favor of the effective string tension (3.4) being given by the D-string tension divided by $n_{5}$. 


\subsection{The $\xi_{i}$ cross-section for $Q_{K}>0$}

The scalar $\xi_{i}$ has the fluctuation equation (2.12) with the effective mass term $M_{\xi}=$ $M_{\lambda-\nu}+M_{+}$. We will try to solve for the cross-section exactly in the regime where $r_{0} \ll$ $r_{n} \ll r_{1}, r_{5}$, so that $T_{R} \ll T_{L}$. We will take $\omega / T_{R}$ to be of order 1 . Hence we should be able to find the dependence of the cross-section on $\omega / T_{R}$, which is a test of the greybody factor dependence.

We will match the approximate solutions in several regions. First, consider the inner region, $r \ll r_{n}$. Here the effective mass is approximately $\frac{8 h}{r^{2}}$, and the equation becomes

$$
\left[h r^{-3} \partial_{r}\left(h r^{3} \partial_{r}\right)+\frac{\omega^{2} r_{1}^{2} r_{5}^{2} r_{n}^{2}}{r^{6}}-\frac{8\left(r^{2}-r_{0}^{2}\right)}{r^{4}}\right] R=0 .
$$

In terms of the variable $z=1-\frac{r_{0}^{2}}{r^{2}}$,

$$
\left[z \partial_{z}\left(z \partial_{z}\right)+\frac{\omega^{2} r_{1}^{2} r_{5}^{2} r_{n}^{2}}{4 r_{0}^{4}}-\frac{2 z}{(1-z)^{2}}\right] R=0
$$

This equation has the same form as (4.2) with

$$
D=\frac{\omega^{2} r_{1}^{4} r_{n}^{2}}{4 r_{0}^{4}}, \quad C=2, \quad E=-2 .
$$

We will again use the substitution (4.3), where now

$$
\begin{aligned}
& E+\beta(\beta-1)=0 \rightarrow(\beta-2)(\beta+1)=0, \\
& \alpha^{2}+D+C+E=0 \rightarrow \alpha=-i \frac{\omega r_{1} r_{5} r_{n}}{2 r_{0}^{2}} .
\end{aligned}
$$

For $r_{0} \ll r_{n}$, we have

$$
T_{R} \approx \frac{r_{0}^{2}}{4 \pi r_{1} r_{5} r_{n}}
$$

Thus,

$$
\alpha=-i \frac{\omega}{8 \pi T_{R}} .
$$

We also choose $\beta=-1$. Hence, the solution is

$$
R_{I}=z^{\alpha}(1-z)^{-1} F(-1+\alpha+i \sqrt{D},-1+\alpha-i \sqrt{D} ; 1+2 \alpha ; z) .
$$

Away from the horizon, i.e. as $z \rightarrow 1$,

$$
R_{I} \rightarrow \frac{r^{2}}{r_{0}^{2}} \frac{2}{1-i \frac{\omega}{4 \pi T_{R}}} \equiv K_{1} \frac{r^{2}}{r_{0}^{2}} .
$$


Now we discuss the region $r_{0} \ll r \ll r_{1}, r_{5}$. Here we may drop the $\omega^{2}$ term, and also set $h=1$. The equation becomes

$$
\left[r^{-3} \partial_{r}\left(r^{3} \partial_{r}\right)-\frac{3 r^{4}+8 r_{n}^{2} r^{2}+8 r_{n}^{4}}{r^{2}\left(r^{2}+r_{n}^{2}\right)^{2}}\right] R=0 .
$$

Substituting $t=1+\frac{r_{n}^{2}}{r^{2}}$, we find

$$
4 \partial_{t}^{2} R-\frac{3-8 t+8 t^{2}}{(1-t)^{2} t^{2}} R=0 .
$$

One may check that the solutions are

$$
[t(t-1)]^{-1 / 2} \quad \text { and } \quad t^{3 / 2}(t-1)^{-1 / 2}(3-2 t) .
$$

To match to the near-horizon solution we choose

$$
R_{I I}=K_{2}[t(t-1)]^{-1 / 2}, \quad K_{2}=\frac{r_{n}^{2}}{r_{0}^{2}} K_{1} .
$$

For large $r$ the solution approaches

$$
R_{I I}=K_{2} \frac{r}{r_{n}} .
$$

In the intermediate region, $r_{n} \ll r \ll 1 / \omega$, we have

$$
\left[r^{-3} \partial_{r}\left(r^{3} \partial_{r}\right)-\frac{3 r_{1}^{4}}{r^{2}\left(r^{2}+r_{1}^{2}\right)^{2}}\right] R=0
$$

with the solution

$$
R_{I I I}=K_{2} \frac{r_{1}}{r_{n}}\left(1+\frac{r_{1}^{2}}{r^{2}}\right)^{-1 / 2} .
$$

In the far region, $r \gg r_{1}$, we have

$$
\left[r^{-3} \partial_{r}\left(r^{3} \partial_{r}\right)+\omega^{2}\right] R=0
$$

with the solution

$$
R_{I V}=2 A(\omega r)^{-1} J_{1}(\omega r) .
$$

Matching the solutions, we find

$$
A=K_{2} \frac{r_{1}}{r_{n}}=K_{1} \frac{r_{1} r_{n}}{r_{0}^{2}} .
$$

The absorption cross-section is given by

$$
\sigma_{\xi}=\frac{4 \pi}{\omega^{3}} \frac{\pi \omega^{3}}{2} r_{1} r_{5} r_{n}|A|^{-2}=\frac{\pi^{2} r_{0}^{4} r_{5}}{2 r_{1} r_{n}}\left(1+\frac{\omega^{2}}{16 \pi^{2} T_{R}^{2}}\right) .
$$

Note that this is exact in $\omega / T_{R}$. To compare this with the effective string result, we take the limit $\omega / T_{L} \rightarrow 0$ in (3.6). Using (4.9) and

$$
T_{L} \approx \frac{r_{n}}{\pi r_{1} r_{5}}
$$

we find exact agreement of the two greybody factors. 


\subsection{The $\eta_{i}$ cross-section for $Q_{K}>0$}

Let us now consider the scalar $\eta_{i}$, which satisfies (2.12) with the effective mass $M_{\eta}=$ $M_{\lambda-\nu}+M_{-}$. We will again solve for the cross-section exactly in the limit $r_{0} \ll r_{n} \ll r_{1}$.

In the inner region, $r \ll r_{n}$, we may ignore the mass term. The equation is

$$
\left[h r^{-3} \partial_{r}\left(h r^{3} \partial_{r}\right)+\frac{\omega^{2} r_{1}^{2} r_{5}^{2} r_{n}^{2}}{r^{6}}\right] R=0
$$

which may be written as

$$
\left[z \partial_{z}\left(z \partial_{z}\right)+\frac{\omega^{2} r_{1}^{2} r_{5}^{2} r_{n}^{2}}{4 r_{0}^{4}}\right] R=0
$$

The solution is

$$
R_{I}=z^{\alpha}
$$

with $\alpha$ given in (4.10). Away from the horizon, i.e. for $z \rightarrow 1, R_{I} \rightarrow 1$.

In the region $r_{0} \ll r \ll r_{1}, r_{5}$ the approximate equation is

$$
\left[r^{-3} \partial_{r}\left(r^{3} \partial_{r}\right)-\frac{3 r^{2}}{\left(r^{2}+r_{n}^{2}\right)^{2}}\right] R=0
$$

which may be recast as

$$
4 \partial_{t}^{2} R-\frac{3}{(t-1)^{2} t^{2}} R=0 .
$$

One may check that the solutions are

$$
t^{3 / 2}(t-1)^{-1 / 2} \quad \text { and } \quad t^{-1 / 2}(t-1)^{-1 / 2}(2 t-1) .
$$

To match to the near horizon solution we pick

$$
R_{I I}=\frac{1}{2} t^{-1 / 2}(t-1)^{-1 / 2}(2 t-1) .
$$

For large $r, R_{I I} \rightarrow \frac{r}{2 r_{n}}$.

In the intermediate region, $r_{n} \ll r \ll 1 / \omega$, the equation is again given by (4.11). The solution matching $R_{I I}$ is

$$
R_{I I I}=\frac{r_{1}}{2 r_{n}}\left(1+\frac{r_{1}^{2}}{r^{2}}\right)^{-1 / 2} .
$$

In the far region, we again find a solution of the form (4.12). Matching the solutions, we find $A=\frac{r_{1}}{2 r_{n}}$. The absorption cross-section is given by

$$
\sigma_{\eta}=2 \pi^{2} r_{1} r_{5} r_{n}|A|^{-2}=8 \pi^{2} \frac{r_{n}^{3} r_{5}}{r_{1}} .
$$

This should be compared with the $\omega / T_{L} \rightarrow 0$ limit of the effective string greybody factor, (3.5). Once again, we find exact agreement! 


\subsection{The Low Temperature limit}

In this section we analyze the $\omega \gg T_{L}, T_{R}$ limit of the greybody factors. In the inner region we ignore the mass term since it is smaller than the $\omega^{2}$ term,

$$
\left[h r^{-3} \partial_{r}\left(h r^{3} \partial_{r}\right)+\frac{\omega^{2} r_{1}^{2} r_{5}^{2}}{r^{4}}\left(1+\frac{r_{n}^{2}}{r^{2}}\right)\right] R=0 .
$$

The solution is

$$
R_{I}=z^{-i(a+b) / 2} F(-i a,-i b, 1-i a-i b, z),
$$

with

$$
a=\frac{\omega}{4 \pi T_{L}}, \quad b=\frac{\omega}{4 \pi T_{R}} .
$$

As $z \rightarrow 1$,

$$
R_{I} \rightarrow E=\frac{\Gamma(1-i a-i b)}{\Gamma(1-i b) \Gamma(1-i a)} .
$$

In the region $r_{1}, r_{5} \gg r \gg r_{n}$, the equation is

$$
\left[r^{-3} \partial_{r}\left(r^{3} \partial_{r}\right)+\frac{\omega^{2} r_{1}^{2} r_{5}^{2}}{r^{4}}-\frac{3}{r^{2}}\right] R=0 .
$$

Substituting $u=\frac{\omega r_{1} r_{5}}{r}$, we get

$$
\left[u \partial_{u}\left(u^{-1} \partial_{u}\right)-1+\frac{3}{u^{2}}\right] R=0
$$

with the solution

$$
R_{I I}=\frac{K_{3}}{r} N_{2}\left(\frac{\omega r_{1} r_{5}}{r}\right) .
$$

To match to the near-horizon solution we first introduce an auxiliary function $\mathcal{R}$ satisfying

$$
\left[r^{-3} \partial_{r}\left(r^{3} \partial_{r}\right)+\frac{\omega^{2} r_{1}^{2} r_{5}^{2}}{r^{4}}\right] \mathcal{R}=0
$$

so that

$$
\mathcal{R}=\frac{\psi}{r} N_{2}\left(\frac{\omega r_{1} r_{5}}{r}\right) .
$$

Matching $\mathcal{R}$ and $R_{I I}$ for small $r$, we find $\psi=K_{3}$. Matching $\mathcal{R}$ and $R_{I}$ for large $r$, we find

$$
\psi=\frac{\pi \omega r_{1} r_{5}}{2} E=K_{3} .
$$

For large $r$,

$$
R_{I I} \rightarrow K_{3} \frac{4 r}{\pi \omega^{2} r_{1}^{2} r_{5}^{2}}
$$


In the intermediate region the equation is again given by (4.11), and now the solution is

$$
R_{I I I}=K_{3} \frac{4}{\pi \omega^{2} r_{1} r_{5}^{2}}\left(1+\frac{r_{1}^{2}}{r^{2}}\right)^{-1 / 2} .
$$

In the far region, $r \gg r_{1}, r_{5}$, the solution is again of the form (4.12). Matching the solutions, we find

$$
A=K_{3} \frac{4}{\pi \omega^{2} r_{1} r_{5}^{2}}=\frac{2}{\omega r_{5}} \frac{\Gamma(1-i a-i b)}{\Gamma(1-i b) \Gamma(1-i a)} .
$$

The absorption cross-section is given by

$$
\sigma_{\mathrm{abs}}=2 \pi^{2} r_{1} r_{5} \sqrt{r_{n}^{2}+r_{0}^{2}}|A|^{-2}=\frac{\pi^{2}}{4} r_{1} r_{5}^{3} r_{0} \cosh \sigma \frac{\omega^{3}}{T_{L}+T_{R}}
$$

where we have used the condition $\omega \gg T_{L}, T_{R}$ to simplify the exponentials. Since the temperatures (3.7) satisfy

$$
T_{L}+T_{R}=\frac{r_{0} \cosh \sigma}{\pi r_{1} r_{5}}
$$

we finally have

$$
\sigma_{\mathrm{abs}}=\frac{\pi^{3}}{4} r_{1}^{2} r_{5}^{4} \omega^{3}
$$

Note that the effective string greybody factors for both scalars, (3.5) and (3.6), exactly agree with this for $\omega \gg T_{L}, T_{R}$. Thus, this is another point of agreement between the semi-classical gravity and the effective string.

\section{Conclusions}

A remarkable feature of the charged supersymmetric black holes is the variety of physically different behaviors exhibited by scalar fields. The minimally coupled and the fixed scalars have been thoroughly analyzed in earlier work, and this paper is devoted to a yet different type of scalars, which we call intermediate.

In the effective string models the physical differences between scalars are due to the different operators they couple to. Indeed, the leading coupling of the minimal scalars is to operators of dimension $(1,1)$, while that of the fixed scalars is to operators of dimension $(2,2)$. In [20] it was observed that the intermediate scalars couple to chiral operators of dimension $(1,2)$ and $(2,1)$. The main achievement of the present work is to discover a surprising mixing between the intermediate scalars from the NS-NS and the R-R sectors. Thus, we find two different intermediate scalars; one of them appears to couple to a dimension $(1,2)$ operator, and the other to a dimension $(2,1)$ operator. In the absence of a momentum flow along the string (the Kaluza-Klein charge), the string theory is non-chiral, and there is no physical difference between these two operators. In this regime we indeed 
find that both intermediate scalars satisfy the same classical equation and, therefore, have identical semi-classical greybody factors, which turn out to agree with the effective string model exactly.

When the momentum flow is present $\left(Q_{K} \neq 0\right)$, the effective string model is chiral, and the two intermediate scalars have different greybody factors, (3.5) and (3.6). Remarkably, now the two classical fluctuation equations (2.12) are different: their near-horizon behavior jumps when $Q_{K}$ is turned on. This jump works in precisely the right way for the semiclassical greybody factors to agree, at least in certain regimes, with the effective string ones. In general we find that, both in the effective string and in the semi-classical approaches, the two intermediate scalars are interchanged by reversing the momentum flow, $Q_{K} \rightarrow-Q_{K}$.

We have tested the greybody factors predicted by the effective string model against the semi-classical calculations in the following regimes,

$$
\begin{array}{ll}
\text { A. } & \frac{\omega}{T_{L}}=\frac{\omega}{T_{R}} \sim O(1) ; \\
\text { B. } & \frac{\omega}{T_{L}} \ll 1, \quad \frac{\omega}{T_{R}} \sim O(1) ; \\
\text { C. } & \frac{\omega}{T_{L}} \gg 1, \quad \frac{\omega}{T_{R}} \gg 1,
\end{array}
$$

finding complete agreement. Unfortunately, we have not been able to extract the semiclassical absorption cross-sections as functions of $\frac{\omega}{T_{L}}$ and $\frac{\omega}{T_{R}}$ in general. For this reason, further analysis of the classical equations (2.12), and comparison with the effective string greybody factors, (3.5) and (3.6), is desirable. This could provide a further sensitive test of the effective string model.

\section{Acknowledgements}

We are grateful to J. Maldacena for useful discussions. The work of I.R.K. was supported in part by DOE grant DE-FG02-91ER40671, the NSF Presidential Young Investigator Award PHY-9157482, and the James S. McDonnell Foundation grant No. 91-48. The work of A.R. was supported in part by the Department of Energy under contract no. DE-AC03-76SF00515. A.A.T. is grateful to the Institute of Theoretical Physics of SUNY at Stony Brook for hospitality during the final stage of this work and acknowledges also the support of PPARC and the European Commission TMR programme grant ERBFMRX-CT96-0045. 


\section{Appendix A. Equations for intermediate scalars: the $D=6$ perspective}

The $D=5$ black hole background (2.3),(2.4) may be viewed as a dimensional reduction of a boosted solitonic black string solution in $D=6$. The equations for small perturbations near this background can thus be derived by expanding the type IIB action reduced to 6 dimensions, assuming that the $D=6$ fluctuations do not depend on the string direction $x^{5}$. This method of derivation clarifies the reason behind the mixing of the fields $A_{5}^{i}$ and $B_{5 i}$. In $D=6$ they appear as the $M=5$ components of the $D=6$ vectors: the KK one, $A_{M}^{i}$, and the RR one, $B_{M i}(i=1,2,3,4 ; M=0,1,2,3,4,5)$. Another conceptual advantage of the $D=6$ approach is that it enables us to include the dependence on the Kaluza-Klein charge, $Q_{K}$, simply by a coordinate transformation (a finite Lorentz boost in the string direction) of the non-extremal case with $Q_{K}=0$.

Let us first consider the $Q_{K}=0$ case. The $D=6$ black string has a trivial dilaton background, $\phi_{6}=0$, so that there is no difference between the Einstein and the string metric. The KK scalar matrix is

$$
G_{i j}=e^{2 \nu} \delta_{i j}, \quad \sqrt{G}=e^{4 \nu},
$$

and the metric is

$$
\begin{aligned}
& d s_{6}^{2}=\left(H_{1} H_{5}\right)^{-1 / 2}\left(-h d t^{2}+d y_{5}^{2}\right)+\left(H_{1} H_{5}\right)^{1 / 2}\left(h^{-1} d r^{2}+r^{2} d \Omega_{4}^{2}\right), \\
& e^{2 \nu}=\left(H_{1} / H_{5}\right)^{-1 / 2}, \quad H_{0 r 5}=2 Q r^{-3} H_{1}^{-2}, \quad \sqrt{g} e^{4 \nu} H^{0 r 5}=2 Q .
\end{aligned}
$$

The R-R antisymmetric tensor field strength, $H_{M N K}$, also has the magnetic (5-brane) components which will not couple to the fluctuation fields we are interested in.

The relevant part of the $D=6$ action that governs small fluctuations of the vector fields $A_{M}^{i}$ and $B_{M i}$, which have trivial background values, is

$$
\begin{gathered}
S_{6}=\frac{1}{2 \kappa_{6}^{2}} \int d^{6} x \sqrt{g}\left[-\frac{1}{4} G_{i j} F_{M N}^{i} F^{j M N}-\frac{1}{4} \sqrt{G} G^{i j} H_{M N i} H_{j}^{M N}-\frac{1}{12} \sqrt{G} H^{M N K} H_{M N K}\right] \\
=\frac{1}{2 \kappa_{6}^{2}} \int d^{6} x \sqrt{g}\left[-\frac{1}{4} e^{2 \nu} F_{i M N} F^{i M N}-\frac{1}{4} e^{2 \nu} H_{M N i} H^{M N i}\right. \\
\left.\quad+\frac{1}{4} e^{4 \nu} H^{M N K}\left(A_{M}^{i} H_{N K i}+B_{M i} F_{N K}^{i}\right)+\ldots\right],
\end{gathered}
$$

where

$$
H_{M N K}=\partial_{M} B_{N K}-\frac{1}{2} A_{M}^{i} H_{N K i}-\frac{1}{2} B_{M i} F_{N K}^{i}+\text { cyclic }, \quad H_{M N i}=\partial_{M} B_{N i}+\text { cyclic . }
$$

4 The crucial point is that the dependence of the non-extremal $D=6$ solution on $Q_{K}$ can be induced by a finite boost, which is not a symmetry of the black string background for $r_{0} \neq 0$. 
Here $B_{M N}$ and $B_{M i}$ are equal to the corresponding components of the $D=10 \mathrm{R}-\mathrm{R} 2$ tensor, up to terms proportional to the $\mathrm{KK}$ vector $A_{M}^{i}$ whose precise form will not be important.

The term linear in $H_{M N K}$ mixes the two vector perturbations in the string background (the two terms in the bracket give equivalent contributions because $H_{M N K}$ has an on-shell value). It is only the electric part of the $H_{M N K}$ background that contributes to the equations for the $A_{5}^{i}, B_{i 5}$ components we are interested in.

It is crucial that the background factors in the kinetic terms for $A_{5}^{i}$ and $B_{i 5}$ are the same. This is a consequence of the R-R nature of $B_{5 i}$ and is not true for its NS-NS counterpart. As a result, one may diagonalize the action introducing

$$
A_{M}^{i+}=\frac{1}{\sqrt{2}}\left(A_{M}^{i}+B_{M i}\right), \quad A_{M}^{i-}=\frac{1}{\sqrt{2}}\left(A_{M}^{i}-B_{M i}\right)
$$

so that the scalars in (2.10) are $A_{5}^{i-}=\xi_{i}, A_{5}^{i+}=\eta_{i}$. The action becomes

$$
\begin{aligned}
S_{6}=S_{+}+S_{-} & =\int d^{6} x \sqrt{g}\left[-\frac{1}{4} e^{2 \nu} F_{M N}^{+i} F^{+i M N}+\frac{1}{4} e^{4 \nu} H^{M N K} A_{M}^{+i} F_{N K}^{+i}\right. \\
& \left.-\frac{1}{4} e^{2 \nu} F_{M N}^{-i} F^{-i M N}-\frac{1}{4} e^{4 \nu} H^{M N K} A_{M}^{-i} F_{N K}^{-i}\right] .
\end{aligned}
$$

Keeping only the relevant electric components we get,

$$
\begin{aligned}
S_{+}= & \int d^{6} x \sqrt{g} e^{2 \nu}\left[\frac{1}{2} F_{0 r}^{+i} F^{+i 0 r}+\frac{1}{2} F_{05}^{+i} F^{+i 05}-\frac{1}{2} F_{5 r}^{+} F^{+i 5 r}\right. \\
& \left.+\frac{1}{2} e^{2 \nu} H^{05 r}\left(A_{5}^{+i} F_{r 0}^{+i}+A_{0}^{+i} F_{5 r}^{+i}+A_{r}^{+i} F_{05}^{+i}\right)\right]
\end{aligned}
$$

and similarly for $S_{-}$. Since we assume that all the fields do not depend on $x^{5}$ (but, in fact, depend only on $r$ and $t$ ), and that the $H_{M N K}$ background is on-shell, the last term is equal to $e^{2 \nu} H^{05 r} A_{5}^{+i} F_{r 0}^{+i}$, up to a total derivative.

$S_{+}$may be viewed as an action for a $2 \mathrm{~d}$ vector $A_{a}^{+}(a=0, r)$ coupled to a scalar $A_{5}^{+i}=\eta_{i}$. To establish a correspondence with the $D=5$ picture, it is natural to integrate out the $(0, r)$ components of the vector $A_{M}^{+}$, which is equivalent in the present context to solving for $F_{r 0}^{+i}$. As a result,

$$
S_{+}=\int d^{6} x \sqrt{g} e^{2 \nu}\left[-\frac{1}{2} \partial_{a} \eta_{i} \partial^{a} \eta_{i}-\frac{1}{2} e^{4 \nu} H^{05 r} H_{05 r} \eta_{i} \eta_{i}\right]
$$

so that we get the scalar equation for $\eta_{i}$ with the mass term determined by the electric part of the $H_{M N K}$ background and originating from the KK vector - R-R vector mixing. 
This is the same mass term for the (rescaled) $\eta_{i}$ as found in the $D=5$ approach of Section 2 for the case of $Q_{K}=0$ (see (2.11),(2.12)).

To find the perturbation equations that include the dependence on the third charge, $Q_{K}$, we may use the fact that the action is invariant under reparametrizations. One may either apply a boost in the $x^{5}$ direction to the background fields or keep the background unchanged, but instead transform the vector components $A_{M}^{ \pm i}, M=0,5$. The boost interpretation of the $Q_{K}$ dependence is manifest in $D=6$ before one integrates out the $0 r$ component of the vector field strength (the boost 'mixes' $F_{0 r}, F_{05}, F_{r 5}$ ). The presence of the $Q_{K}$-dependent cross-term in (2.9) is understood from the $D=6$ perspective to be a consequence of the mixing between $A_{M}^{i}$ and $B_{M i}$ occurring already for $Q_{K}=0$, and of the fact that a non-zero boost creats an extra term in the action which is linear in $F_{05}$. As a result, one finds the same equations (2.12) as obtained in the $D=5$ approach. 


\section{References}

[1] A. Strominger and C. Vafa, Phys. Lett. B379 (1996) 99, hep-th/9601029.

[2] C.G. Callan and J. Maldacena, Nucl. Phys. B472 (1996) 591, hep-th/9602043.

[3] G. Horowitz and A. Strominger, Phys. Rev. Lett. 77 (1996) 2368, hep-th/9602051.

[4] G. Horowitz, J. Maldacena and A. Strominger, Phys. Lett. B383 (1996) 151, hepth/9603109.

[5] J. Maldacena and L. Susskind, Nucl. Phys. B475 (1996) 670, hep-th/9604042.

[6] S.R. Das and S.D. Mathur, Nucl. Phys. B478 (1996) 561, hep-th/9606185.

[7] A. Dhar, G. Mandal and S.R. Wadia, Phys. Lett. B388 (1996) 51, hep-th/9605234.

[8] S.S. Gubser and I.R. Klebanov, Nucl. Phys. B482 (1996) 173, hep-th/9608108.

[9] S.R. Das and S.D. Mathur, Nucl. Phys. B482 (1996) 153, hep-th/9607149.

[10] J. Maldacena and A. Strominger, Phys. Rev. D55 (1997) 861, hep-th/9609026.

[11] S.S. Gubser and I.R. Klebanov, Phys. Rev. Lett. 77 (1996) 4491, hep-th/9609076.

[12] E. Keski-Vakkuri and P. Krauss, hep-th/9610045.

[13] I.R. Klebanov and S.D. Mathur, hep-th/9701187.

[14] S. Hawking and M. Taylor-Robinson, hep-th/9702045.

[15] F. Dowker, D. Kastor and J. Traschen, hep-th/9702109.

[16] J. Maldacena and A. Strominger, hep-th/9702015.

[17] I.R. Klebanov, hep-th/9702076.

[18] S.S. Gubser, I.R. Klebanov and A.A. Tseytlin, hep-th/9703040.

[19] B. Kol and A. Rajaraman, hep-th/9608126.

[20] C.G. Callan, Jr., S.S. Gubser, I.R. Klebanov and A.A. Tseytlin, Nucl. Phys. B489 (1997) 65, hep-th/9610172; I.R. Klebanov and M. Krasnitz, Phys. Rev. D55 (1997) 3250, hep-th/9612051.

[21] I.R. Klebanov and M. Krasnitz, hep-th/9703216.

[22] J. Maharana and J.H. Schwarz, Nucl. Phys. B390 (1993) 3, hep-th/9207016.

[23] A.A. Tseytlin, Mod. Phys. Lett. A11 (1996) 689, hep-th/9601177.

[24] M. Cvetič and D. Youm, Nucl. Phys. B476 (1996) 118, hep-th/9603100.

[25] J. Maldacena, Nucl. Phys. B477 (1996) 168, hep-th/9605016. 